\title{
Dispersion of surface plasmon polaritons on short-pitch metal gratings
}

\author{
I. R. Hooper and J. R. Sambles \\ Thin Film Photonics Group, School of Physics, University of Exeter, Stocker Road, Exeter EX4 4QL, United Kingdom
}

(Received 21 August 2001; published 12 April 2002)

\begin{abstract}
The dispersion of surface plasmon polaritons (SPPs) has been calculated for short-pitch metal gratings for various depths. For gratings with depths greater than their pitch very flat SPP bands are formed in the zero-order region of the spectrum which may be resonantly excited with radiation polarized with its electric field in the plane of incidence of the radiation, which also contains the grating vector. The dispersion curves of these modes evolve as deformations of the familiar shallow grating dispersion curve due to the opening of very large band gaps, and interactions of the SPP bands with both the light line and other SPP bands. Also presented are the dispersion curves for the equivalent modes excited by radiation having its plane of incidence perpendicular to the grating vector, but polarized with its electric field parallel to this grating vector. The full dispersion curve of these SPP bands for all orientations of the grating relative to the plane of incidence is also presented.
\end{abstract}

DOI: $10.1103 /$ PhysRevB.65.165432

PACS number(s): 78.20.-e

\section{INTRODUCTION}

It has long been understood that when a thick metal layer is patterned with a grating structure the resulting surface may no longer act as a simple mirror due first to diffraction from the corrugation, and second to the excitation of surface plasmon polaritons (SPPs). ${ }^{1,2,3}$ However, until recently it had been assumed that if the corrugation has a pitch less than half the wavelength of the incident radiation (in other words, it is zero order, or nondiffractive) then the interface would once again act as a good mirror. The reason for this is that, as well as having no available diffracted orders, it was thought that SPPs would no longer be excited since, even with the added momentum available to the incident radiation due to scattering from the grating, the momentum of SPPs on such a structure is greater than the photon momentum available to the radiation in the zero-order region of the spectrum. However, for very deep zero-order monogratings the SPP dispersion curve may be so severely modified from the flat surface case that resonant absorption of light due to SPP excitation may occur within the zero-order region of the spectrum. ${ }^{4,5}$ These self-coupled SPP resonances are flat banded, possessing near zero group velocity over a large range of incident wave vectors (Fig. 1).

The majority of work on the SPP band structures of metal gratings relates to low-aspect ratio structures for which results may be explained using perturbation theory. ${ }^{6}$ However, recent advances in the manufacture of submicron structures has enabled the fabrication of high-aspect ratio grating structures which support highly localized resonances within the grooves. ${ }^{7,8}$ These flat-banded resonances are very different to SPPs on shallow gratings, and have been explained as being due to hybrid waveguide-SPP resonances. However the evolution of these bands as a function of groove depth was not investigated and has not been explained in any detail. An extension of the studies of deep gratings, conducted by Porto, Garcia-Vidal, and Pendry ${ }^{9}$ evaluated the band structures for lamellar transmission gratings with a pitch of 3.5 $\mu \mathrm{m}$ and a depth of $4 \mu \mathrm{m}$. This also showed flat-banded resonances in the infrared region of the spectrum and predicted almost total resonant transmittance of the incident light in the zero-order region of the spectrum. Other recent developments have shown that resonant-enhanced optical transmission can take place through hole arrays in metal films, ${ }^{10,11}$ which is also associated with SPP excitation. ${ }^{12}$

This paper intends to bridge the gap between the wellunderstood band structure of SPPs on shallow gratings and those of the high-aspect ratio structures recently investigated, concentrating on the zero-order region of the spectrum. It will also extend the previous work of Tan et al. ${ }^{5}$ to explore the SPP band structure for zero-order gratings at orientations other than that of the grooves perpendicular to the plane of incidence of the exciting electromagnetic (EM) radiation.

A SPP is a fundamental electromagnetic surface mode at a metal/dielectric interface consisting of a surface chargedensity oscillation (the surface plasmon) coupled to the exciting EM radiation. By solving Maxwell's equations for such a planar interface the dispersion of these modes can be obtained and is described by

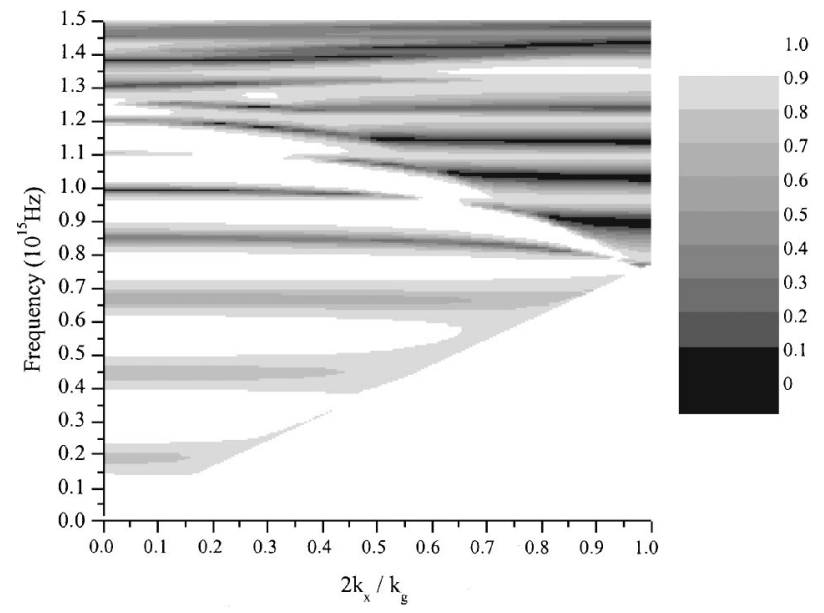

FIG. 1. Reflectivity as a function of frequency and in-plane wave vector for TM-polarized light on a $300 \mathrm{~nm}$ deep, $50 \mathrm{~nm}$ wide Gaussian-grooved, $200 \mathrm{~nm}$ pitch silver grating held at a zero degree azimuthal angle. The bands are very flat for a large range of incident wave vectors. 


$$
k_{\mathrm{spp}}=k_{0}\left(\frac{\varepsilon_{1} \varepsilon_{2}}{\varepsilon_{1}+\varepsilon_{2}}\right)^{1 / 2},
$$

where $k_{\text {spp }}$ is the wave vector of the SPP, $k_{0}$ is the wave vector of the incident radiation, and $\varepsilon_{1}$ and $\varepsilon_{2}$ are the frequency-dependent dielectric functions of the two media on either side of the interface. This equation describes the familiar flat-surface SPP dispersion curve which starts at the light line and curves away as $k_{x}$ is increased to approach $\omega_{\mathrm{sp}}$ (the surface plasmon frequency) asymptotically. For a single planar surface this SPP dispersion curve is always beyond the light line in $\omega-k$ space, and therefore cannot be directly coupled to by incident light.

When a monograting structure is added it has the affect of folding the bands at the Brillouin-zone boundaries set up by the grating periodicity which results in the SPP dispersion curve being brought into a region of $\omega$ - $k$ space where it may be coupled to by incident radiation. If the pitch of the grating is small enough that the grating is zero order for visible light $\left(\lambda_{g}<200 \mathrm{~nm}\right)$ then even the folded back (diffracted) SPP dispersion curve of a shallow grating is always at higher $\omega$ than that of the visible light and no SPP is excitable. This is, however, no longer the case as the depth of the grating is increased.

Section III of this paper will be concerned with the band structure of these zero-order monograting structures for radiation incident such that the grating vector is parallel to the plane of incidence.

\section{THE MODELING CODE}

The modeling method used in this paper has already been reported elsewhere ${ }^{13}$ and is based upon a method originally proposed by Chandezon et al., in which a nonorthogonal curvilinear coordinate transformation is used to map the grating profile onto a flat plane. ${ }^{14}$ This enables easier matching of the tangential components of the fields at the boundaries, from which a scattering matrix is determined. By identifying the poles of the scattering matrices in the $\omega-k$ plane the dispersion curves for any modes available to the system can be plotted. The reflection and transmission coefficients may also be evaluated from the scattering matrices.

In all of the following work the permittivity of the metal gratings is modeled as that of silver, being described by a Drude model with a plasma frequency of $\omega_{p}=1.32$ $\times 10^{16} \mathrm{~s}^{-1}$, and a relaxation time of $\tau=1.45 \times 10^{-14} \mathrm{~s}$.

\section{BAND GAPS AND THE FORMATION OF SELF-COUPLED SPPS ON SHORT-PITCH METAL GRATINGS}

When two branches of the SPP dispersion curve cross at the Brillouin-zone boundaries created by the periodicity there are two counter propagating SPPs which interact to produce two standing wave solutions. The two solutions have different field distributions with respect to the surface corrugation, and these correspond to different energies thereby producing a band gap. ${ }^{15,16}$

The size of the band gap is largely determined by the

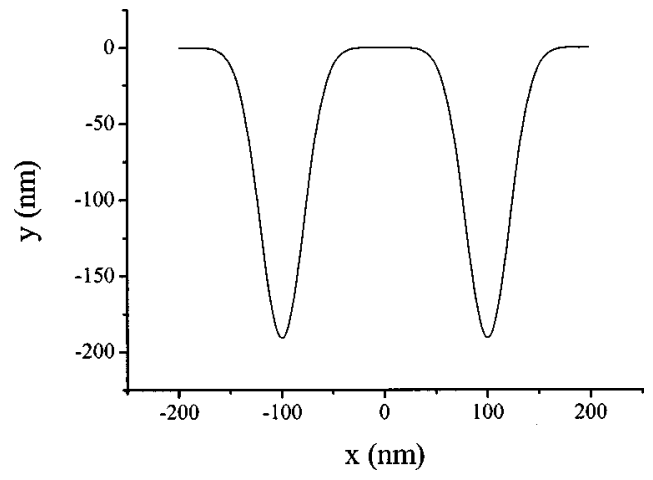

FIG. 2. An example of the type of grating structure investigated. The profile is made up of a series of Gaussian grooves so that the depth, width, and pitch of the grating may be altered independently, and so that in deep structures the SPP modes in neighboring grooves will be largely isolated from each other.

depth of the grating, with deeper gratings giving larger band gaps. However, the relative coupling strength to the two branches at normal incidence is mainly determined by the shape of the grating profile.

If the grating is described by a finite Fourier series of the form

$$
f(x)=a_{0} \sin \left(k_{g} x+\phi_{1}\right)+a_{1} \sin \left(2 k_{g} x+\phi_{2}\right)+\cdots
$$

then it is scattering from the first harmonic $\left(a_{1}\right)$ which determines the coupling strength to the two branches. If the phase of the first harmonic is $+90^{\circ}$ relative to the fundamental then the lower branch is coupled to strongly, whereas if the phase is $-90^{\circ}$ then coupling to the higher-energy branch predominates.

In the modeling presented in this paper this coupling difference between the two bands at normal incidence does not appear in the scattering matrix calculations since this does not involve the coupling between the surface modes and the exciting radiation. However, it does show in the calculated reflectivities since it relates to the symmetry of the exciting radiation fields with respect to the grating surface. Because any applications which may make use of these SPP resonances will involve excitation with EM radiation, it is important to note that, as well as the band structures of these modes, these coupling effects determine the optical response of the grating.

If a grating which has a large first harmonic with a phase of $+90^{\circ}$ is investigated then a band structure which shows a large band gap with strong coupling to the lower-energy branch is produced. An example of such a structure is a Gaussian groove profile, and it is this structure which is investigated in the following work in this section (Fig. 2). It is also used because previous work has shown the SPP resonances on deep Gaussian-grooved gratings to be highly localized within the grooves. The use of Gaussian grooves on a grating of $200 \mathrm{~nm}$ pitch reduces interactions between neighboring grooves, allowing the band structure of more highly localized modes to be obtained.

Figure 3 shows the band structure, in the grating vector direction, for the first Brillouin zone of a $200 \mathrm{~nm}$ pitch silver 
(a)

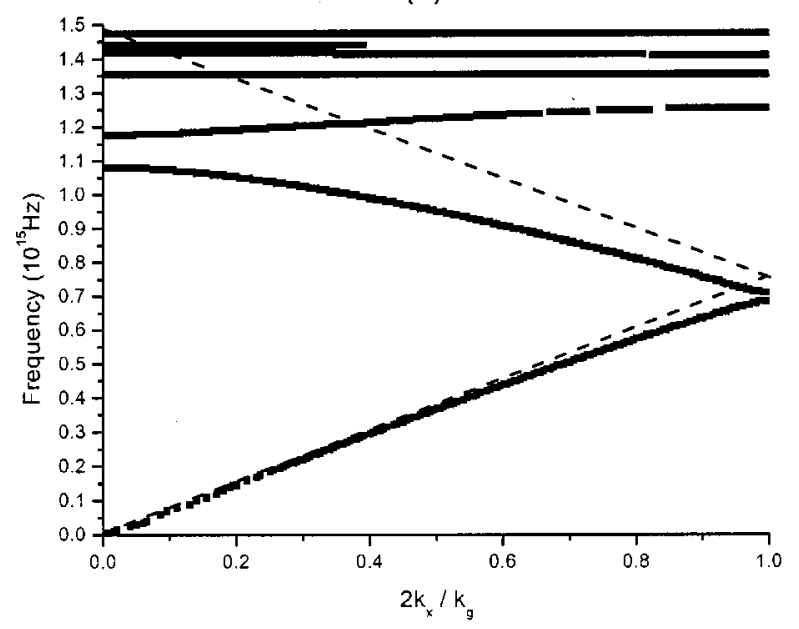

(c)

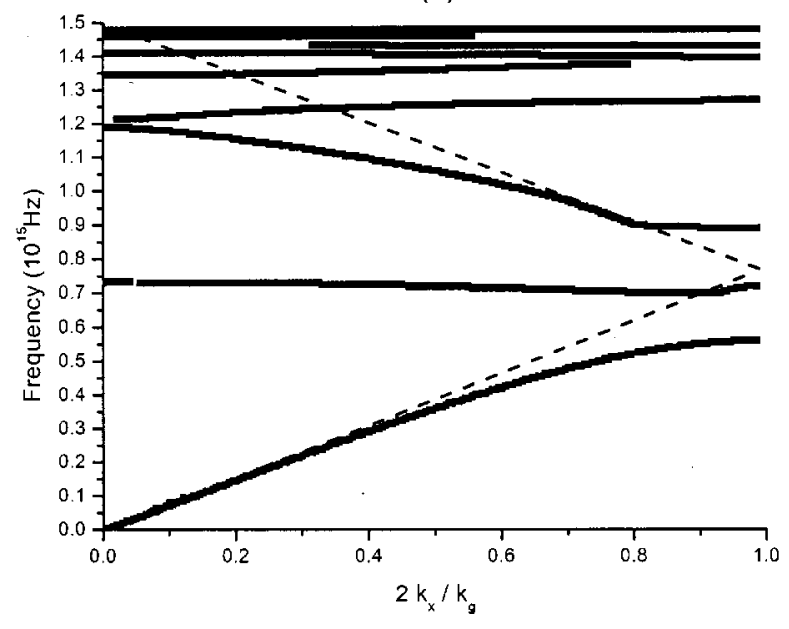

(b)

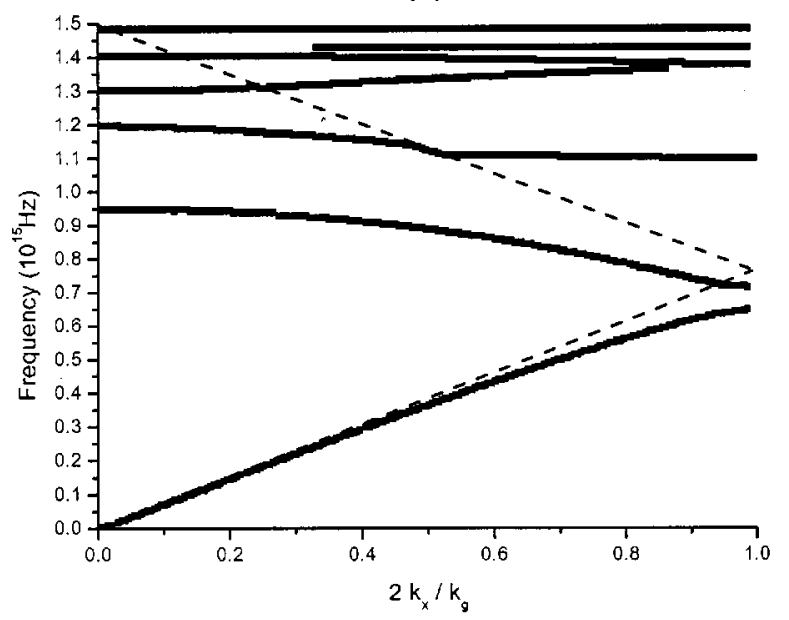

(d)

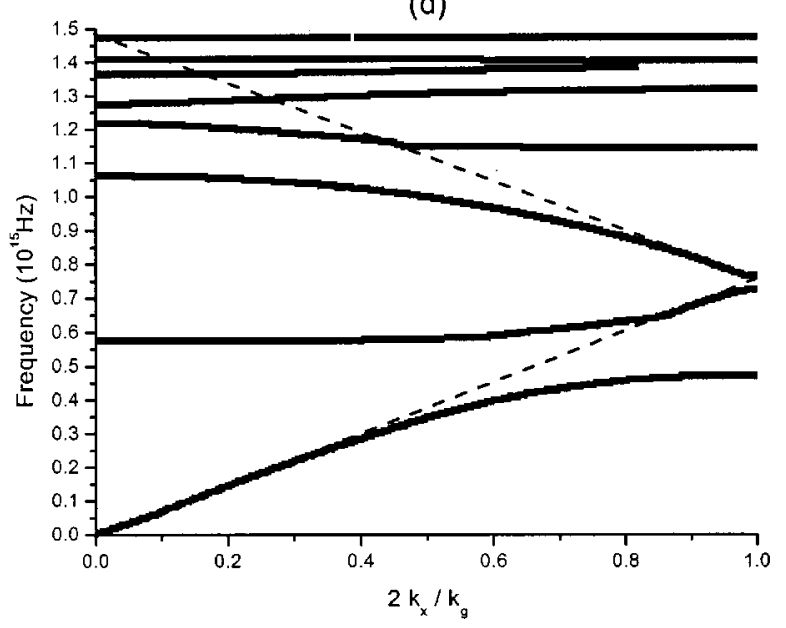

FIG. 3. Dispersion curves for $200 \mathrm{~nm}$ pitch, $50 \mathrm{~nm}$ wide, Gaussian-grooved silver gratings for $k_{z}=0$, with (a) $d=10 \mathrm{~nm}$, (b) $d$ $=25 \mathrm{~nm}$, (c) $d=50 \mathrm{~nm}$, and (d) $d=75 \mathrm{~nm}$.

grating with $50 \mathrm{~nm}$ wide Gaussian grooves for 10, 25, 50, and $75 \mathrm{~nm}$ depths. In Fig. 3(a) the band structure looks very similar to that of a shallow sinusoidal grating. However, due to the large first harmonic needed to create the Gaussian profile, relatively large band gaps are opened up (for example at between 1.1 and $1.2 \times 10^{15} \mathrm{~Hz}$ at $k_{x}=0$ ).

When the depth is increased these band gaps widen with the low-energy solutions reducing in energy until, when the depth is $50 \mathrm{~nm}$ [Fig. 3(c)], a very flat band is forming. On further increase of depth to $75 \mathrm{~nm}$ [Fig. 3(d)] this branch, while remaining quite flat, has a positive gradient close to the light line, while the second order low-energy branch has passed through the first-order high-energy branch and is in the process of forming a second flat band. The formation of these flat bands will be discussed in more detail later.

To understand the nature of these resonances it is instructive to investigate their field profiles. Figure 4 shows the $\left|\mathbf{H}_{z}\right|$ ( $z$ is along the groove direction) component of the fields at $k_{x}=0$ for the low-energy branch of the second-order transverse magnetic (TM) resonance for $10 \mathrm{~nm}(f=1.36$ $\left.\times 10^{15} \mathrm{~Hz}\right), 25 \mathrm{~nm}\left(f=1.3 \times 10^{15} \mathrm{~Hz}\right)$ and $50 \mathrm{~nm}(f=1.19$ $\left.\times 10^{15} \mathrm{~Hz}\right)$ deep gratings. For the resonance on a relatively shallow $10 \mathrm{~nm}$ deep grating field maxima are observed on the tops of the grating between the grooves and also at the bottoms of the grating grooves. In addition to these there are two extra field maxima per grating period found on the grating groove "shoulders." (This arises because the secondorder SPP at $k_{x}=0$ corresponds to a standing wave created by $\pm 2 k_{g}$ scattering, thus the wave has four field amplitude maxima per grating period). When the depth is increased to $25 \mathrm{~nm}$ the fields on the opposing shoulders of a groove appear to couple together, and by a depth of $50 \mathrm{~nm}$ these coupled fields are almost entirely localized within the grating grooves. Since the fields of the SPPs on either side of the groove have coupled together these resonances can be described as self-coupled SPPs (SCSPPs).

The dispersion curves for 100, 150, and $300 \mathrm{~nm}$ deep gratings are shown in Fig. 5. These show how the opening of even larger band gaps have pushed the low-energy branches of the first-, second-, and third-order SPPs into the zero-order region of the spectrum. Indeed, the first-order branch has been moved beyond the visible to the infrared region of the spectrum. It is also noticeable that the character of the bands very much resembles that expected from anticrossing of flat bands with the light line. In fact, in previous work, ${ }^{5}$ it has been predicted that for deep lamellar gratings a series of 
(a)

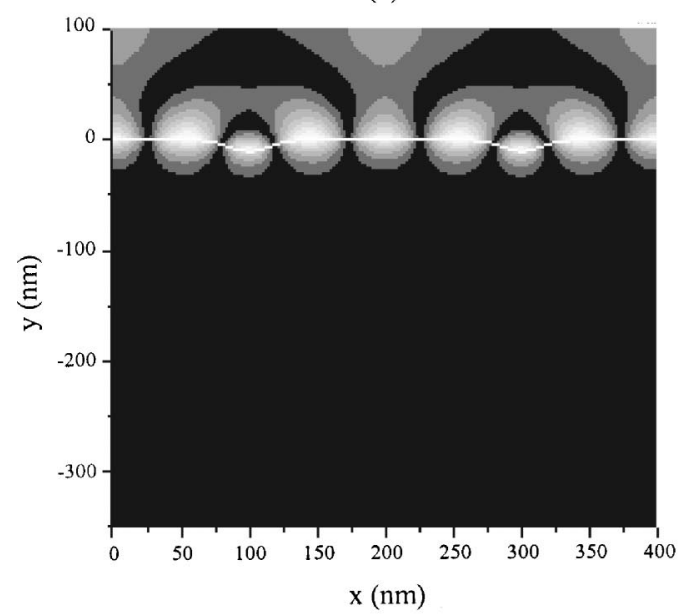

(b)

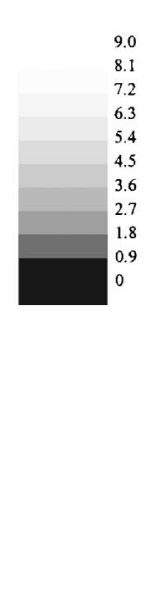

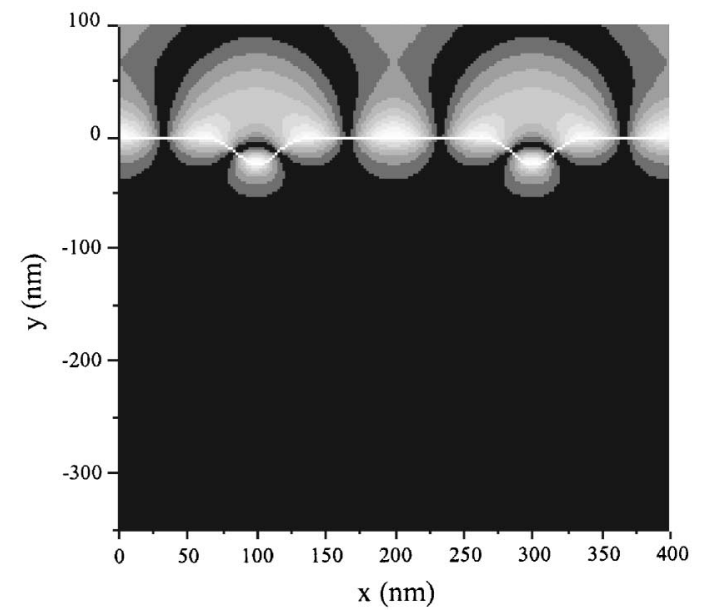

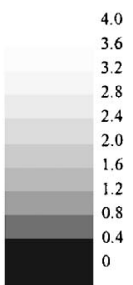

(c)
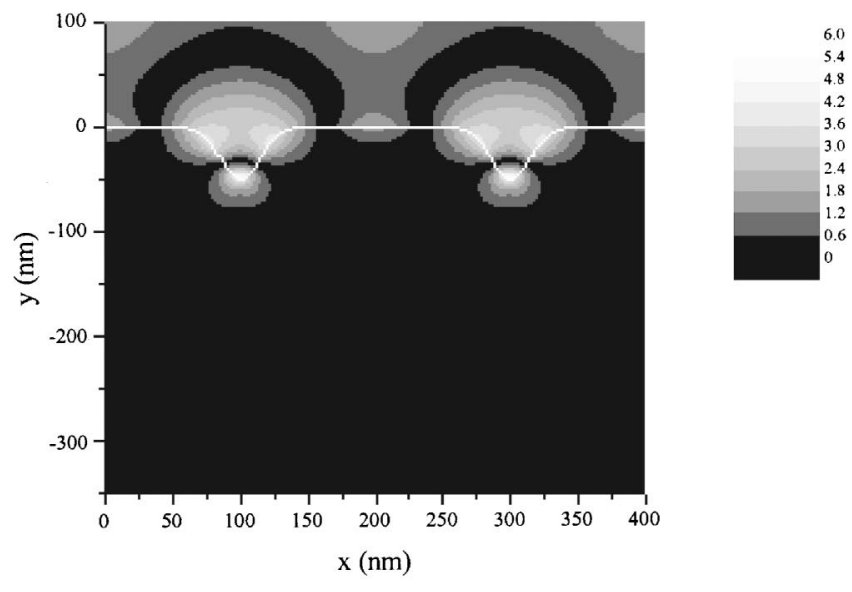

FIG. 4. $\left|\mathbf{H}_{z}\right|$ for the second-order SPP resonance for (a) $d=10 \mathrm{~nm}, f=1.36 \times 10^{15} \mathrm{~Hz}$, (b) $d=25 \mathrm{~nm}, f=1.3 \times 10^{15} \mathrm{~Hz}$, and (c) $d$ $=50 \mathrm{~nm}, f=1.19 \times 10^{15} \mathrm{~Hz}$.

almost flat bands with a small negative gradient would be expected. However, these flat bands interact with the free radiation and, since the density of states of the free radiation becomes divergent at grazing incidence, this leads to strong anticrossing between the flat bands and the light line as shown.

The dispersion curve for the $300 \mathrm{~nm}$ deep grating [Fig. $5(\mathrm{c})]$ shows several quite flat bands within the radiative regime. The $\left|\mathbf{H}_{z}\right|$ component of the fields for successive flat bands at $k_{x}=0$ are shown in Fig. 6 . These show that there are a family of localized SCSPP resonances whose number of field maxima contained within the grating grooves is equal to the order of the mode which correspond to the value of $k_{g}$ from which the branch originates. The fields on the flat regions of the dispersion curves for $k_{x}$ values away from the symmetry points of $k_{x}=0$ and the Brillouin-zone boundaries show similar field distributions to those observed at normal incidence, the principle difference being the intensity distribution of the fields. These intensity differences vary periodically along the grating, with the periodicity being determined by the ratio of $2 k_{x} / k_{g}$ [e.g., at $2 k_{x} / k_{g}=0.5$ the fields in alternate grooves are the same (Fig. 7)].
The nature of these resonances, and the mechanism by which they arise on short pitch gratings has been discussed. However, there are other aspects to the formation of these bands, and in order to discuss these it is useful to investigate the position of the SPPs in frequency as a function of depth (Fig. 8). At $k_{x}=0$ [Fig. 8(a)] the first-order SPP (starting at $f \approx 1.15 \times 10^{15} \mathrm{~Hz}$ ) shows a band-gap opening as the depth is increased. The splitting is highly asymmetric with the lowenergy branch reducing in frequency rapidly with increasing depth, and the high-energy mode changing comparatively little. In order to understand this asymmetry it is necessary to consider the coupling of the surface plasmon with the free radiation.

Earlier the SPP dispersion curve for a planar metal interface was described as a solution of Maxwell's equations. However, Maxwell's equations implicitly contain the coupling of the free radiation to the SPP, and it is this coupling which creates the familiar flat-surface dispersion curve, rather than a straight line at $\omega_{\mathrm{sp}}$. When a grating structure is added and band gaps are opened at the Brillouin zone boundaries, the high-energy branches cannot increase in frequency above the light line (or its corresponding diffracted light line 
(a)

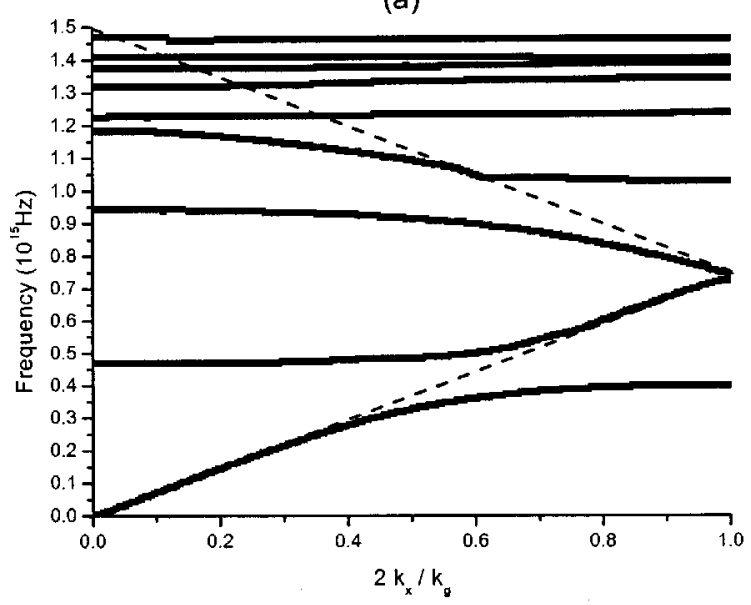

(b)

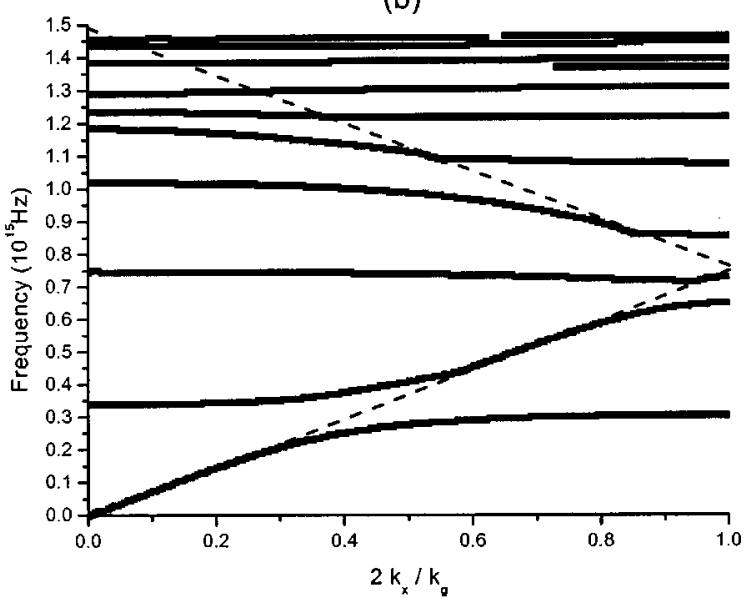

(c)

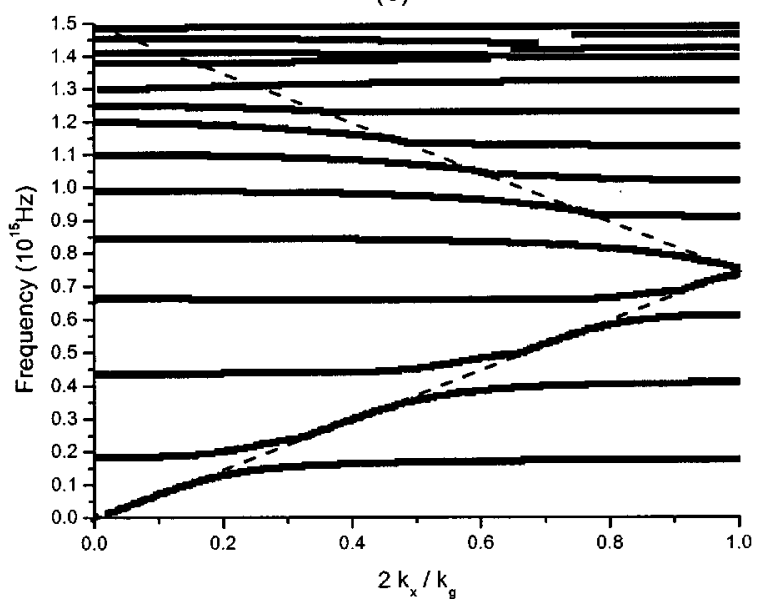

FIG. 5. Dispersion curves for $200 \mathrm{~nm}$ pitch, $50 \mathrm{~nm}$ wide, Gaussian-grooved silver gratings for $k_{z}=0$, with (a) $d=100 \mathrm{~nm}$, (b) $d$ $=150 \mathrm{~nm}$, and (c) $d=300 \mathrm{~nm}$.

in the reduced zone scheme) since the coupling to the free radiation prevents it. For this reason the opening of the band gap is asymmetric; when the depth of the grating is increased the low-energy branch may more readily reduce in energy relative to the high-energy branch, which may only increase in energy up to that of the light line, increasing asymptotically towards it with increasing depth.

When the band gaps increase at $k_{x}=0$ the higher-order low-energy branches are free to pass through the lower-order high-energy branches with no interaction since this is at a symmetry point in $\omega-k$ space. At large depths the frequency at which these low-energy SPP branches may be excited reduces as approximately $1 / d$. This can be simply predicted by considering the resonances as standing-wave modes within the grooves.

As $k_{x}$ is increased anticrossing between higher-order lowenergy branch SPPs and lower-order high-energy branch SPPs arises because the symmetry of the system has been broken. This can clearly be observed in Fig. 8(b) $\left(2 k_{x} / k_{g}\right.$ $=0.1)$. At $2 k_{x} / k_{g}=0.5$ the anticrossing is so strong that the branches are no longer well defined [Fig. 8(c)], and it is also noticeable that the coupling of the SPP bands with the free radiation causes them to tend towards the light line (dotted lines at $f=0.38 \times 10^{15} \mathrm{~Hz}$ and $1.14 \times 10^{15} \mathrm{~Hz}$ ). This is because of the interaction of the SPP bands with the light line.

In Fig. $7(\mathrm{~d})\left(2 k_{x} / k_{g}=1.0\right.$, at the Brillouin-zone boundary $)$ there is no longer any anticrossing between the high- and low-energy SPP bands since it is another symmetry point in $\omega-k$ space. However, anticrossing between the low-energy branches and the light line at $f=0.75 \times 10^{15} \mathrm{~Hz}$ is still evident.

\section{SELF-COUPLED SURFACE PLASMON POLARITONS EXCITED WITH TE POLARIZED RADIATION}

So far only the band structure in the $\omega-k_{x}$ plane with $k_{z}$ $=0$ (a $0^{\circ}$ azimuthal angle) has been discussed. In this orientation only TM-polarized radiation can be used to excite the SCSPP modes since a component of the radiation $E$ field must be perpendicular to the surface for excitation to occur. However, at normal incidence the case at $0^{\circ}$ azimuth with TM-polarized radiation is the same as for TE polarized radiation at an azimuthal angle of $90^{\circ}$. Therefore any SPP resonance excitable for one case should be excitable for the other. This is found to be the case, while the dispersion of these modes in the $\omega$ - $k_{z}$ plane with $k_{x}=0$ is significantly 
(a)

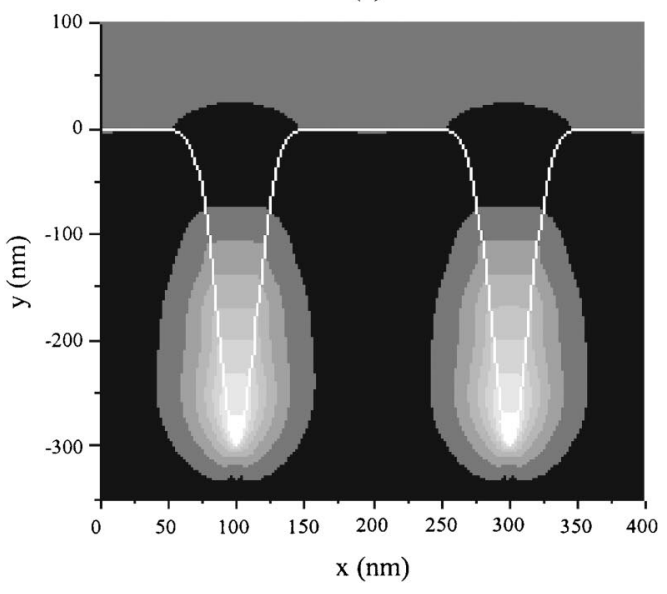

(c)

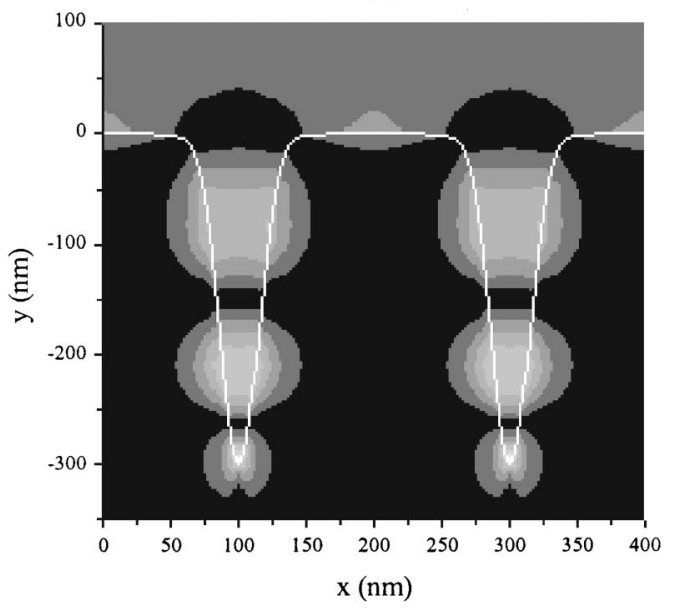

(b)
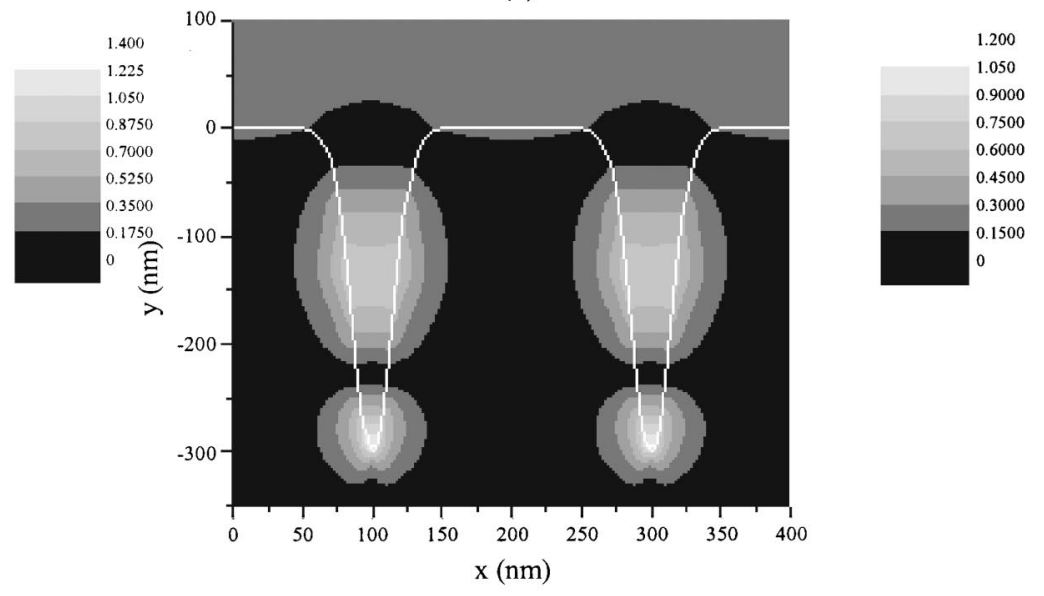

(d)
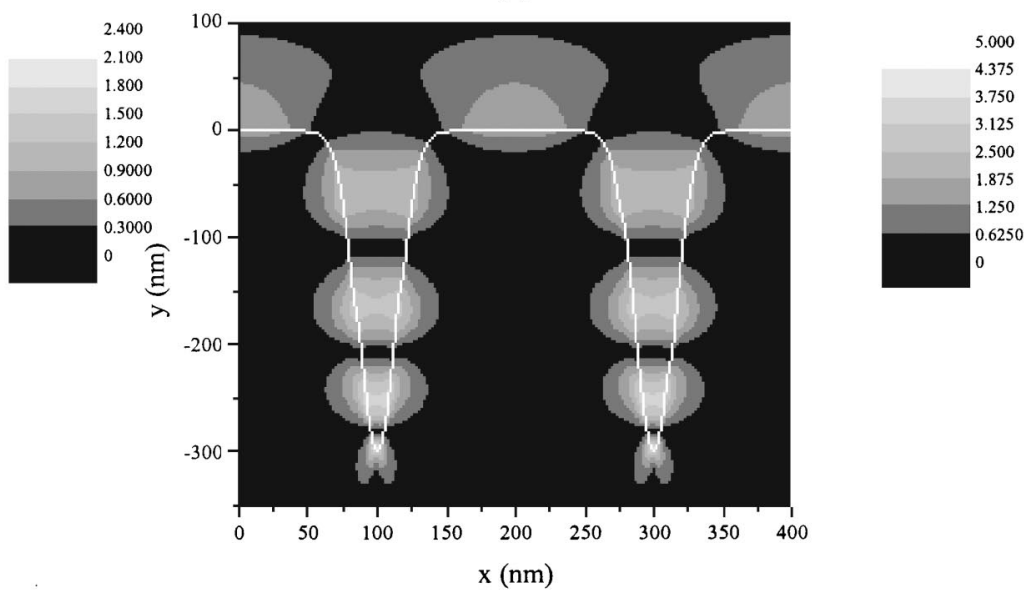

FIG. 6. $\left|\mathbf{H}_{z}\right|$ for the first four SCSPPs on a $300 \mathrm{~nm}$ deep, $50 \mathrm{~nm}$ wide Gaussian-grooved grating with $k_{z}=0$. (a) $f=0.19 \times 10^{15} \mathrm{~Hz}$, (b) $f=0.44 \times 10^{15} \mathrm{~Hz}$, (c) $f=0.66 \times 10^{15} \mathrm{~Hz}$, and (d) $f=0.85 \times 10^{15} \mathrm{~Hz}$.

different from those in the other orientation (the $\omega$ - $k_{x}$ plane with $k_{z}=0$ ).

Figure 9 shows the dispersion curve along the $k_{z}$ direction for the TE polarized resonances on a $300 \mathrm{~nm}$ deep Gaussian-
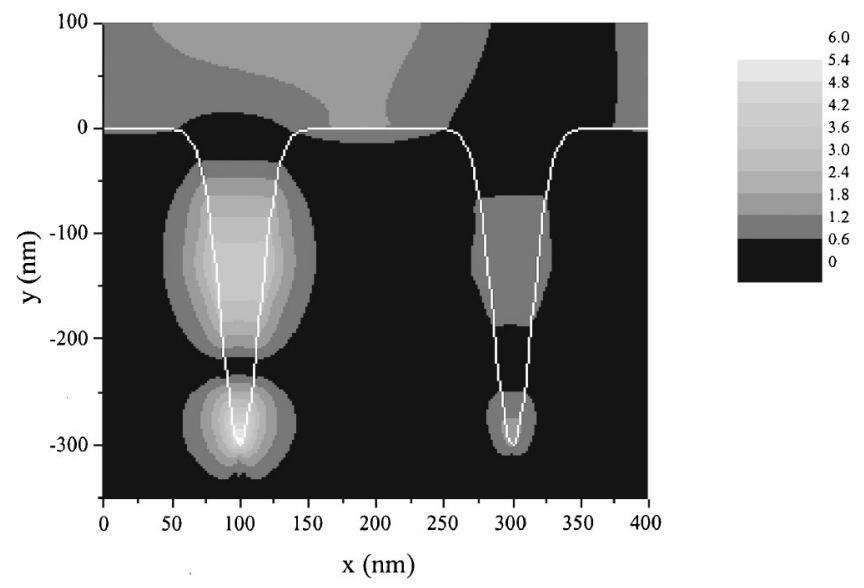

FIG. 7. $\left|\mathbf{H}_{z}\right|$ for the second-order SCSPP resonance on a $300 \mathrm{~nm}$ deep, $50 \mathrm{~nm}$ wide Gaussian-grooved grating with $2 k_{x} / k_{g}=0.5(f$ $\left.=0.46 \times 10^{15} \mathrm{~Hz}\right)$. grooved grating. There are three different features noticeable on this plot. First, the diagonal line starting at the origin is the light line. Moving away from this as $k_{z}$ is increased is a curve which originates at the origin and is the flat surface SPP curve (slightly deformed by the grating in the $x$ direction). Third, there are a series of bands which at normal incidence occur at the same frequencies as the TM-excited resonances at normal incidence. These bands, though relatively flat, gently curve up in energy and, outside of the light line, approach the grating-perturbed flat surface SPP curve. To understand the dispersion of these modes it is necessary to consider the SPP curve which has been scattered from the grating.

Previously the flat-surface SPP dispersion curve has been considered only in the $k_{x}$ direction. Of course it also disperses in the $k_{z}$ direction, and when this is considered the dispersion curve appears as a horn shape centerd at the origin and flattening out asymptotically to $\omega_{\mathrm{sp}}$ in all directions. When a grating is added in the $x$-direction folding of the SPP, bands at the Brillouin-zone boundaries causes the horn shape to fold into the region of $\omega-k$ space available to incident radiation. Another way of considering this band folding is to position the grating-deformed SPP curves at integer values of 
(a)

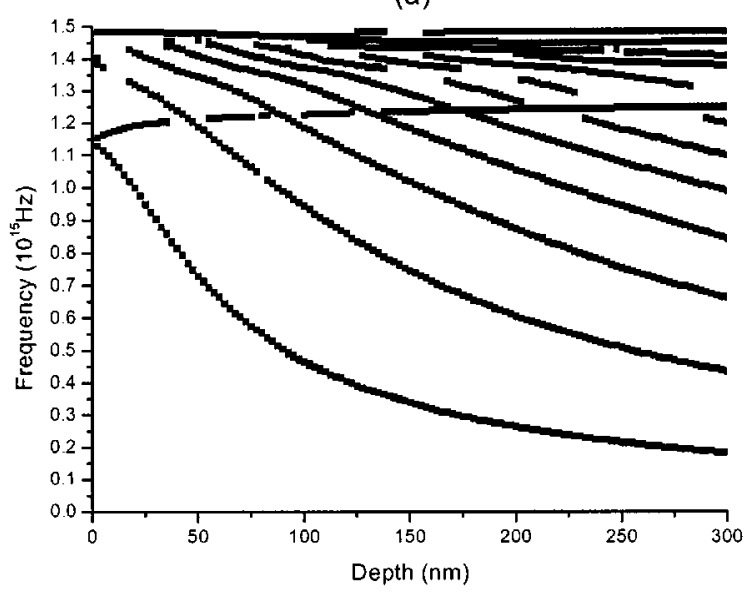

(c)

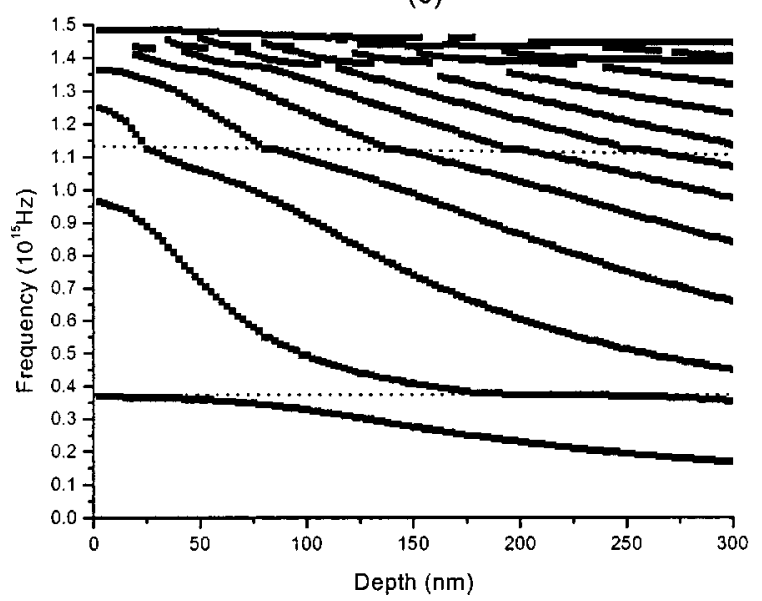

(b)

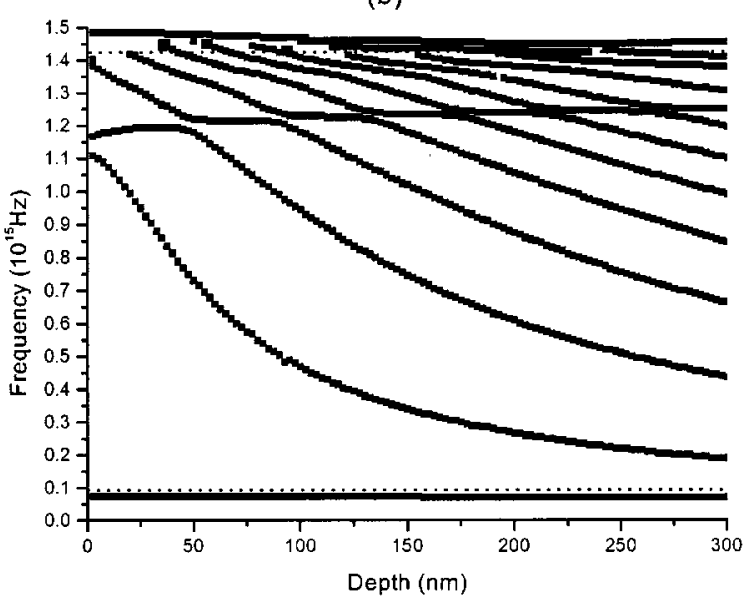

(d)

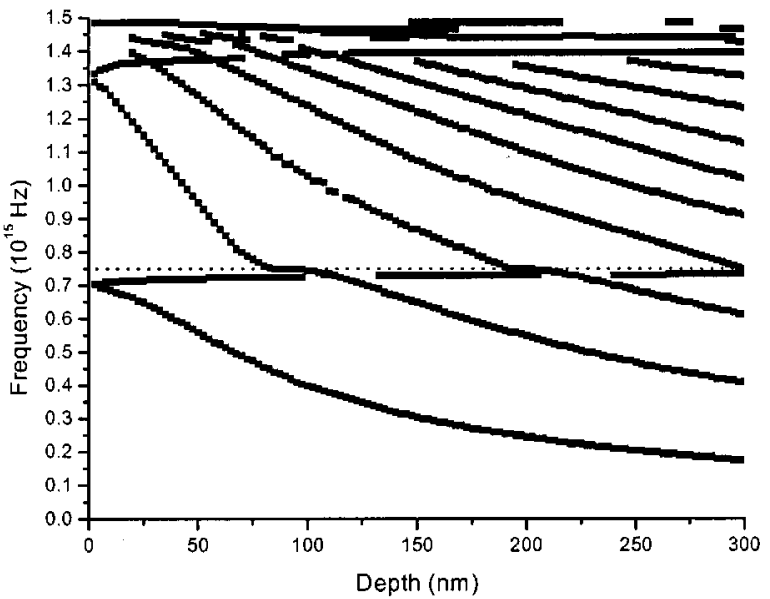

FIG. 8. Mode frequency as a function of grating depth for $200 \mathrm{~nm}$ pitch, $50 \mathrm{~nm}$ wide, Gaussian-grooved gratings at $k_{z}=0$. (a) $2 k_{x} / k_{g}$ $=0.0$, (b) $2 k_{x} / k_{g}=0.1$, (c) $2 k_{x} / k_{g}=0.5$, and (d) $2 k_{x} / k_{g}=1.0$. Dotted lines are light lines.

the grating vector in the $x$ direction to represent the scattering of the SPP from the grating surface. For a very shallow grating, where there is little deformation of the SPP dispersion curve, a slice in $\omega-k$ space through the plane at $k_{x}=0$

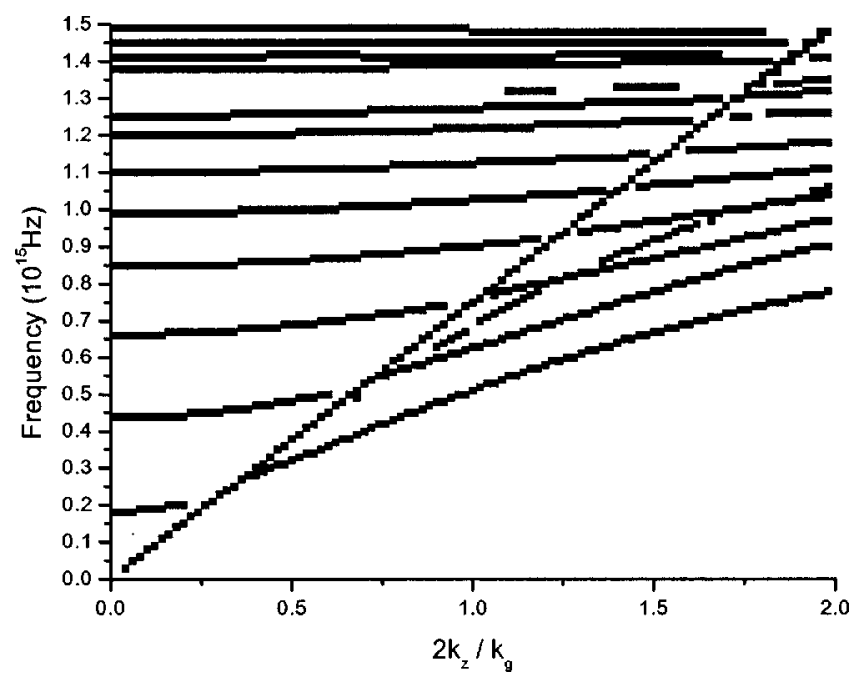

FIG. 9. The dispersion curve for a $200 \mathrm{~nm}$ pitch, $300 \mathrm{~nm}$ deep, $50 \mathrm{~nm}$ wide Gaussian-grooved grating with $k_{x}=0$ and varying $k_{z}$. will show the three types of features in Fig. 9. The light line is present, as is the SPP horn centerd at the origin. But there is also a curve which represents a slice through the scattered SPP curve centerd at $k_{x}=k_{g}$. This is a band which rises in energy as $k_{z}$ is increased and approaches the SPP curve centerd at the origin at large values of $k_{z}$. Of course, at $k_{x}=0$ two SPPs scattered from the grating are actually interacting; one scattered from $+k_{g}$, and one from $-k_{g}$. Therefore, the mode is a standing wave in the $x$ direction, which propagates in the $z$ direction.

For a deep grating the band gaps and anticrossing effects cause the SPP curve to deform in the $x$ direction as discussed previously. However, the effect of taking a slice through the scattered SPP curve in the $\omega-k_{z}$ plane at $k_{x}=0$ produces bands which at normal incidence occur at a frequency defined by the deformation of the SPP dispersion curve caused by the grating. As $k_{z}$ is increased the effect of the grating structure diminishes since the SPP is no longer propagating perpendicular to the grating grooves. This reduces the size of the band gaps causing the bands to curve up in frequency until at large $k_{z}$ values they approach the dispersion curve centered at the origin.

It is noticeable in Fig. 9 that, unlike the dispersion of these SCSPP bands in the $k_{x}$ direction, there is no coupling 


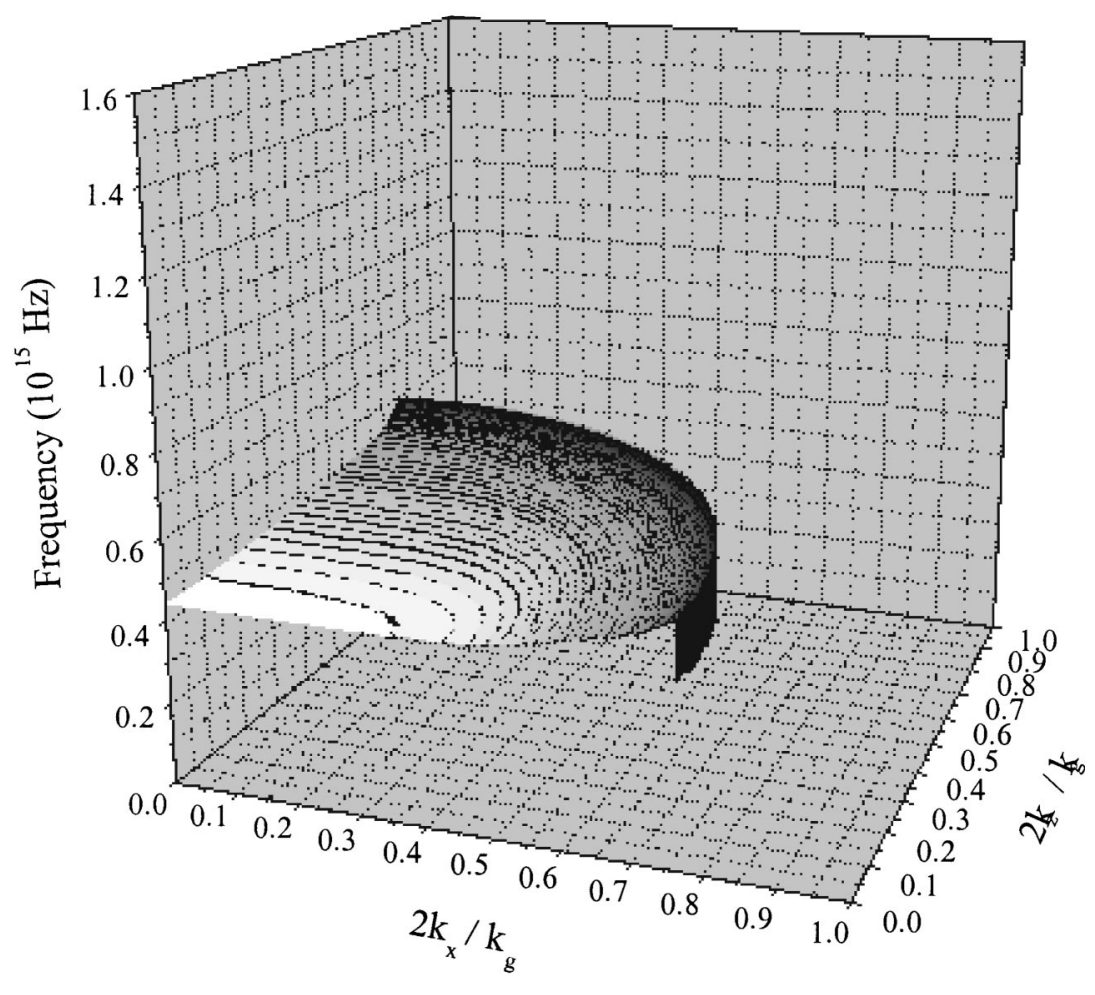

FIG. 10. The frequency of maximum absorption due to the excitation of the second-order SCSPP on a $200 \mathrm{~nm}$ pitch, $300 \mathrm{~nm}$ deep, $50 \mathrm{~nm}$ wide Gaussian-grooved grating for varying $k_{x}$ and $k_{z}$. with the free radiation at the light line. In the case of TMpolarized radiation incident in the $\omega-k_{x}$ plane at $k_{z}=0$ there is a periodic variation of the electric field in the direction of the grating vector when $k_{x}$ is nonzero. Because of this the overlap integral of the wave functions of the incident light and the SCSPP mode will also be nonzero resulting in an interaction between them. By contrast, for TE-polarized light incident in the $\omega-k_{z}$ plane at $k_{x}=0$ there is no spatially periodic variation in the incident electric field in the direction of the grating vector for any values of $k_{z}$. Therefore, the overlap integral is zero and there can be no interaction. This is a special case and only occurs when $k_{x}=0$. At all other orientations, where there is a finite $k_{x}$, coupling of the SPP mode with the free radiation can take place, and splitting occurs.

\section{BAND STRUCTURE FOR A SCSPP BAND FOR ALL GRATING ORIENTATIONS}

By calculating the absorption due to the excitation of a SCSPP resonance with both TM- and TB-polarized incident radiation it is possible to trace the SCSPP band for all possible grating orientations within the light line (the absorption is used as opposed to the loss of reflection to avoid the influence of polarization conversion from the grating). The absorption (due to Joule heating in the metal) was calculated as a function of frequency for varying $k_{x}$ and $k_{z}$ within the light line. By finding the peaks in the absorption the frequencies at which resonant excitation of the SCSPP occurs may be found, and by plotting these the dispersion of a SCSPP band for all orientations of the grating with respect to the incident radiation may be obtained.

This has been performed for the second-order SCSPP resonance on a $300 \mathrm{~nm}$ deep Gaussian-grooved grating, and the results are shown in Fig. 10. For the planes at $k_{x}=0$, and $k_{z}=0$, the curves have the forms described in the previous sections, and for values of $k_{x}$ and $k_{z}$ between these planes the band structure is seen to vary smoothly. In the $\omega-k_{x}$ plane at $k_{z}=0$ this band can only be excited with TM-polarized radiation, and in the $\omega-k_{z}$ plane at $k_{x}=0$ only TE-polarized radiation can excite it. For finite values of both $k_{x}$ and $k_{z}$ both polarizations can excite the mode, however the coupling strength will vary due to the magnitude of the $E$-field component perpendicular to the surface of the grating structure changing as the grating orientation with respect to the incident radiation is changed.

The fact that there is a relatively flat band producing absorption for all grating orientations could be useful for such application as selective absorbers. However, its use may be limited because the structure will only absorb particular polarizations at certain grating orientations. The use of a bigrating structure could overcome this limitation and produce a structure which will absorb the same frequencies of the incident light for all polarizations at all azimuthal angles, and for a wide range of polar angles.

\section{CONCLUSIONS}

The form of the dispersion of a family of flat-banded selfcoupled surface plasmon polaritons (SCSPPs) on very deep zero-order gratings at $k_{z}=0$ has been explored. Their band structure has been described by considering the deformation of the familiar shallow grating SPP dispersion curve caused by the large depth of the structure. Very large band gaps have been opened and, along with anticrossing of the SCSPP bands with the light line, and anticrossing between SPP bands away from the Brillouin-zone boundaries, these have been shown to produce the dispersion curves obtained from 
the modeling. Also, the equivalent modes in the $\omega-k_{z}$ plane at $k_{x}=0$, which are excitable with TE-polarized radiation, have been shown to exist and are a product of the interaction of two SPPs scattered from $\pm k_{g}$ producing a SPP which is a standing wave in the $x$ direction (parallel to the grating vector) but which may propagate in the $z$ direction (parallel to the grating grooves). Finally, the dispersion of a single band of these modes has been calculated for all possible orientations of the grating with respect to the incident radiation, showing that a relatively flat-banded mode can be excited for all azimuthal angles.

These flat bands may be of interest in selective absorbers or in resonant-enhanced Raman scattering. They may also be of use in coupling radiation out of fluorescent species situated within the grating grooves.

\section{ACKNOWLEDGMENTS}

The authors are grateful for EPSRC support and the provision of a CASE award by QinetiQ (Farnborough) for I. R. Hooper. They also acknowledge helpful discussions with Dr. C. R. Lawrence, Dr. A. P. Hibbins, and Dr. W. C. Tan. This work was carried out as part of Technology Group 08 of the MoD Corporate Research Fund.
${ }^{1}$ R. W. Wood, Proc. R. Soc. London A 18, 269 (1902).

${ }^{2}$ U. Fano, J. Opt. Soc. Am. 31, 213 (1941).

${ }^{3}$ H. Raether, Surface Plasmons (Springer-Verlag, Berlin, 1988).

${ }^{4}$ M. B. Sobnack, W. C. Tan, N. P. Wanstall, T. W. Preist, and J. R. Sambles, Phys. Rev. Lett. 80, 5667 (1998).

${ }^{5}$ W. C. Tan, T. W. Preist, J. R. Sambles, and N. P. Wanstall, Phys. Rev. B 59, 12661 (1999).

${ }^{6}$ A. A. Maradudin, in Surface Polaritons, edited by V. M. Agranovich and D. L. Mills (North-Holland, New York, 1982), p. 405.

${ }^{7}$ T. Lopez-Rios, D. Mendoza, F. J. Garcia-Vidal, J. SanchezDehesa, and B. Pannetier, Phys. Rev. Lett. 81, 665 (1998).

${ }^{8}$ F. J. Garcia-Vidal, J. Sanchez-Dehesa, A. Dechelette, E. Bustarret, T. Lopez-Rios, T. Fournier, and B. Pannetier, J. Lightwave Technol. 17, 2191 (1999).

${ }^{9}$ J. A. Porto, F. J. Garcia-Vidal, and J. B. Pendry, Phys. Rev. Lett.
83, 2845 (1999).

${ }^{10}$ T. W. Ebbesen, H. J. Lezec, H. F. Ghaemi, T. Thio, and P. A. Wolff, Nature (London) 391, 667 (1998).

${ }^{11}$ H. F. Ghaemi, Tineke Thio, D. E. Grupp, T. W. Ebbesen, and H. J. Lezec, Phys. Rev. B 58, 6779 (1998).

${ }^{12}$ L. Martin-Moreno, F. J. Garcia-Vidal, H. J. Lezec, K. M. Pellerin, T. Thio, J. B. Pendry, and T. W. Ebbesen, Phys. Rev. Lett. 86, 1114 (2001).

${ }^{13}$ N. P. K. Cotter, T. W. Preist, and J. R. Sambles, J. Opt. Soc. Am. 12, 1097 (1995).

${ }^{14}$ J. Chandezon, M. T. Dupuis, G. Cornnet, and D. Maystre, J. Opt. Soc. Am. 72, 839 (1982).

${ }^{15}$ W. L. Barnes, T. W. Preist, S. C. Kitson, J. R. Sambles, N. P. Cotter, and D. J. Nash, Phys. Rev. B 51, 11164 (1995).

${ }^{16}$ W. L. Barnes, T. W. Preist, S. C. Kitson, and J. R. Sambles, Phys. Rev. B 54, 6227 (1996). 